PRL-TH-94/08

TIFR/TH/94-07

\title{
CONSTRAINTS ON BARYON-NONCONSERVING YUKAWA COUPLINGS IN A SUPERSYMMETRIC THEORY
}

\author{
Biswajoy Brahmachari ${ }^{(\mathrm{a})}$ and Probir Roy ${ }^{(\mathrm{b})}$ \\ (a) Theory Group, Physical Research Laboratory, Navrangpura, \\ Ahmedabad 380 009, INDIA, biswajoy@prl.ernet.in \\ (b) Theoretical Physics Group, Tata Institute of Fundamental Research, \\ Homi Bhabha Road, Bombay 400 005, INDIA, probir@tifrvax.bitnet
}

PACS number(s) : 11.30.Pb, 12.10.Dm

\begin{abstract}
$\underline{\text { Abstract }}$
The 1-loop evolution of couplings in the minimal supersymmetric standard model, extended to include baryon nonconserving $(\not B)$ operators through explicit $R$-parity violation, is considered keeping only $B$ superpotential terms involving the maximum possible number of third generation superfields. If all retained Yukawa couplings $Y_{i}$ are required to remain in the perturbative domain $\left(Y_{i}<1\right)$ upto the scale of gauge group unification, upper bounds ensue on the magnitudes of the $B$ coupling strengths at the supersymmetry breaking scale, independent of the model of unification. They turn out to be similar to the corresponding fixed point values reached from a wide range of $Y_{i}$ (including all $Y_{i}$ greater than unity) at the unification scale. The coupled evolution of the top and $B$ Yukawa couplings results in a reduction of the fixed point value of the former.
\end{abstract}

\footnotetext{
${ }^{1}$ Address after 1st October 1994: High Energy Section, International Center For Theoretical Physics, P.O. Box 586, 34100 Trieste, Italy.
} 
There is widspread interest today in baryon number violating $(B)$ processes. Many studies have been made of $B$ couplings in the context of baryogenesis, proton decay etc. Yet, one of the least investigated sources of baryon nonconservation is the set of $\not B$ operators which occur in the superpotential of the minimal supersymmetric standard model (MSSM) extended to include explicit $R$-parity breaking terms [1].

In this paper we start with all such operators coming from the superpotential. We know from the Standard Model that Yukawa coupling strengths spectacularly reflect the generation hierarchy. The third generation ones are the strongest - followed by those of the second generation with the first generation couplings being the weakest. It would be reasonable to adopt a similar hypothesis for the $\not B$ Yukawa couplings and retain only those which are the strongest - discarding the rest. Because of color antisymmetry, not all three superfields in the trilinear superpotential term can belong to the third generation. We deem it sufficient to retain only those terms in which the third generation superfields appear twice. We bound the magnitudes of the corresponding coupling strengths from above by utilizing constraints from Renormalization Group Evolution (RGE). The bounds result from the requirement that all Yukawa couplings in the theory remain in the perturbative domain upto the scale of the unification of gauge couplings [2] $M_{U} \sim 2 \times 10^{16} \mathrm{GeV}$.

$R$-parity $R_{p} \equiv(-)^{3 B+L+2 S}$ (with $B, L, S$ as baryon number, lepton number and spin respectively) distinguishes between particles $\left(R_{p}=1\right)$ and sparticles $\left(R_{p}=-1\right)$. The popular formulation [3] of the MSSM has $R_{p^{-}}$ 
conservation built into it by fiat. Many recent studies suggest [4] nonetheless $\not R_{p}$ couplings, allowed by renormalizability and supersymmetry, that admit both $B$ and $L$ violation. However, the simultaneous presence of both types of terms and the (naturalness-based) supposition that the supersymmetry breaking scale $M_{S U S Y}$ should be $\lesssim 0(\mathrm{TeV})$ would imply catastrophic proton decay unobserved in nature. This forces practitioners of explicit $\not t_{p}$ models to consider either lepton or baryon nonconserving cases.

Thus two $R_{p}$ scenarios are in vogue. There is one with purely lepton number violating interactions, respecting baryon number:

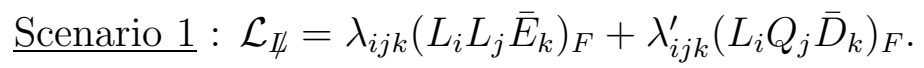

The second $\not R_{p}$ secnario has purely baryon number violating terms with conserved lepton number:

Scenario $2: \mathcal{L}_{\beta}=\lambda_{i j k}^{\prime \prime}\left[\bar{D}_{i} \bar{D}_{j} \bar{U}_{k}\right]_{F}$

Here $\lambda, \lambda^{\prime}$ and $\lambda^{\prime \prime}$ are Yukawa couplings with $\lambda^{\prime}$ and $\lambda^{\prime \prime}$ being antisymmetric in $i, j$. Moreover, $L, Q, \bar{E}, \bar{D}, \bar{U}$ stand for the doublet lepton, doublet quark, singlet antilepton, singlet $d$-antiquark, and singlet $u$-antiquark superfields respectively, with subscripts acting as generation indices.

Either (1) or (2) has been shown [4] to be consistent with some deeper fundamental theory and therefore can be seriously entertained. Moreover, cosmological bounds on $\lambda, \lambda^{\prime}$ and $\lambda^{\prime \prime}$, once believed to be strong [5], have been shown [6] to be not generally valid. In particular, a GUT era leptogenesis makes $\lambda_{i j k}^{\prime \prime}$ completely free [6] of cosmological constraints. Serious 
phenomenological upper bounds do exist [7], of course, on the magnitudes of most of the $\lambda$ and $\lambda^{\prime}$ components from the nonobservation of various leptonic rare decays, neutrino Majorana mass etc. Comparatively, the $\lambda^{\prime \prime}-$ components, apart from $\lambda_{211}^{\prime \prime}$ and $\lambda_{311}^{\prime \prime}[8]$ which have been strongly bounded from the lack of any observed $n \bar{n}$ oscillation, stand relatively unconstrained. It is therefore worthwhile to try to derive some upper bounds, however weak, on the magnitudes of the remaining $\lambda^{\prime \prime}$ components from the requirement of a perturbative behaviour. We also investigate the fixed point values of the top and $(B)$ Yukawa couplings. We discover that the former is somewhat reduced in strength from its value in the $R_{p}$ conserving case while the latter are comparable to the above-mentioned bounds.

RGE is our basic tool in constraining the couplings of all superfields $\Phi^{a}$. Here $a$ is a generic index. Given a trilinear term $d_{a b c} \Phi^{a} \Phi^{b} \Phi^{c}$ in the superpotential and the evolution scale $\mu$, the RGE equation for $d_{a b c}$ is [9]

$$
\mu \frac{\partial}{\partial \mu} d_{a b c}=\gamma_{a}^{i} d_{i b c}+\gamma_{b}^{j} d_{a j c}+\gamma_{c}^{k} d_{a b k} .
$$

In (3) $\gamma_{a}{ }^{i}$ is the anomalous dimension matrix $Z_{-1 / 2}^{-1} \mu \partial / \partial \mu Z_{k}^{1 / 2}{ }_{k}^{i}$ where the renormalization constant $Z$ relates the renormalized superfield $\Phi$ and the unrenormalized one $\Phi_{0}$ by

$$
\Phi_{0}{ }^{i}=Z_{a}^{1 / 2}{ }_{a}^{i} \Phi^{a} .
$$

We would apply (3) to the Yukawa couplings of interest. The Yukawa part 
of the Lagrangian density including $\left(\not P, \not R_{p}\right)$ terms is $[1]$ :

$\mathcal{L}=h_{\tau}\left[L_{3} H_{1} \bar{E}_{3}\right]_{F}+h_{b}\left[Q_{3} H_{1} \bar{D}_{3}\right]_{F}+h_{t}\left[Q_{3} H_{2} \bar{U}_{3}\right]_{F}+\lambda_{233}^{\prime \prime}\left[\bar{D}_{2} \bar{D}_{3} \bar{U}_{3}\right]_{F}+\lambda_{133}^{\prime \prime}\left[\bar{D}_{1} \bar{D}_{3} \bar{U}_{3}\right]_{F}$

As explained earlier, we include only the terms with the maximum possible number of third generation indices in the $\not B$ part of $\mathcal{L}$. Of course, we also have the three regular Yukawa terms coming from the third generation (characterized by the couplings $h_{t}, h_{b}, h_{\tau}$ ) and ignore their lower generation counterparts. In (4) $H_{1}$ and $H_{2}$ are the Higgs superfields coupling to the up and down quarks respectively.

\begin{tabular}{|c|c|c|}
\hline $\begin{array}{c}\text { SUPERFIELDS } \\
\Phi\end{array}$ & $\begin{array}{c}\text { REPRESENTATIONS } \\
S U(3)_{L} \times S U(2)_{L} \times U(1)_{Y}\end{array}$ & $\begin{array}{c}\text { ANOMALOUS DIMENSION } \\
(4 \pi)^{2} \gamma_{\Phi}^{\Phi}\end{array}$ \\
\hline$L_{3}$ & $(1,2,-1 / 2)$ & $h_{\tau}^{2}-\frac{3}{2} g_{2}^{2}-\frac{3}{10} g_{Y}^{2}$ \\
$\bar{E}_{3}$ & $(1,1,1)$ & $2 h_{\tau}^{2}-\frac{6}{5} g_{Y}^{2}$ \\
$\bar{D}_{3}$ & $(\overline{3}, 1,1 / 3)$ & $2 h_{b}^{2}+6 \lambda_{233}^{\prime \prime 2}+6 \lambda_{133}^{\prime \prime 2}-\frac{8}{3} g_{c}^{2}-\frac{2}{15} g_{Y}^{2}$ \\
$\bar{D}_{2}$ & $(\overline{3}, 1,1 / 3)$ & $6 \lambda_{233}^{\prime \prime 2}-\frac{8}{3} g_{c}^{2}-\frac{2}{15} g_{Y}^{2}$ \\
$\bar{D}_{1}$ & $(\overline{3}, 1,1 / 3)$ & $6 \lambda_{133}^{\prime \prime 2}-\frac{8}{3} g_{c}^{2}-\frac{2}{15} g_{Y}^{2}$ \\
$\bar{U}_{3}$ & $(\overline{3}, 1,-2 / 3)$ & $2 h_{t}^{2}+6 \lambda_{133}^{\prime \prime 2}+6 \lambda_{233}^{\prime \prime 2}-\frac{8}{3} g_{c}^{2}-\frac{8}{15} g_{Y}^{2}$ \\
$Q_{3}$ & $(3,2,1 / 6)$ & $h_{b}^{2}+h_{t}^{2}-\frac{8}{3} g_{c}^{2}-\frac{3}{2} g_{2}^{2}-\frac{1}{30} g_{Y}^{2}$ \\
$H_{1}$ & $(1,2,-1 / 2)$ & $h_{\tau}^{2}+3 h_{b}^{2}-\frac{3}{2} g_{2}^{2}-\frac{3}{10} g_{Y}^{2}$ \\
$H_{2}$ & $(1,2,1 / 2)$ & $3 h_{t}^{2}-\frac{3}{2} g_{2}^{2}-\frac{3}{10} g_{Y}^{2}$ \\
\hline
\end{tabular}

Table 1 : Properties of the relevent superfields.

The $S U(3)_{C} \times S U(2)_{L} \times U(1)_{Y}$ representations and the anomalous dimen- 
sions $\gamma_{\Phi}^{\Phi}$ of the superfields (diagonal elements of the anomalous dimension matrix), occurring in (3), are given in Table 1 (N.B. $Q=I_{3}+Y$ ). The B terms mix different generations at the 1-loop level. In particular, they lead to nonzero values of the following off-diagonal terms in the anomalous dimension matrices:

$$
\begin{aligned}
\gamma_{\bar{D}_{1}} & =\frac{3}{8 \pi^{2}} \lambda_{133}^{\prime \prime} \lambda_{233}^{\star \prime \prime}, \\
\gamma_{\bar{D}_{2}} & =\frac{3}{8 \pi^{2}} \lambda_{133}^{\star \prime \prime} \lambda_{233}^{\prime \prime} .
\end{aligned}
$$

All other off-diagonal elements vanish.

We can now write down the evolution equations of the Yukawa couplings. (Soft supersymmetry breaking terms have dimensional strengths and they do not contribute to the evolution of dimensionless couplings.) The equations are

$$
\begin{aligned}
& \mu \frac{\partial}{\partial \mu} h_{\tau}=h_{\tau}\left(\gamma_{L_{3}}^{L_{3}}+\gamma_{H_{1}}^{H_{1}}+\gamma_{\bar{E}_{3}}^{\bar{E}_{3}}\right), \\
& \mu \frac{\partial}{\partial \mu} h_{b}=h_{b}\left(\gamma_{Q_{3}}^{Q_{3}}+\gamma_{H_{1}}^{H_{1}}+\gamma_{\bar{D}_{3}}^{\bar{D}_{3}}\right), \\
& \mu \frac{\partial}{\partial \mu} h_{t}=h_{t}\left(\gamma_{\bar{U}_{3}}^{\bar{U}_{3}}+\gamma_{H_{2}}^{H_{2}}+\gamma_{\bar{D}_{3}}^{\bar{D}_{3}}\right), \\
& \mu \frac{\partial}{\partial \mu} \lambda_{133}^{\prime \prime}=\lambda_{133}^{\prime \prime}\left(\gamma_{\bar{U}_{3}}^{\bar{U}_{3}}+\gamma_{\bar{D}_{3}}^{\bar{D}_{3}}+\gamma_{\bar{D}_{1}}^{\bar{D}_{1}}\right)+\lambda_{233}^{\prime \prime} \gamma_{\bar{D}_{1}}^{\bar{D}_{2}}, \\
& \mu \frac{\partial}{\partial \mu} \lambda_{233}^{\prime \prime}=\lambda_{233}^{\prime \prime}\left(\gamma_{\bar{U}_{3}}^{\bar{U}_{3}}+\gamma_{\bar{D}_{3}}^{\bar{D}_{3}}+\gamma_{\bar{D}_{2}}^{\bar{D}_{2}}\right)+\lambda_{133}^{\prime \prime} \gamma_{\bar{D}_{2}}^{\bar{D}_{1}} .
\end{aligned}
$$

Next, we define $t=1 /(2 \pi) \ln (\mu / \mathrm{GeV})$ and, utilizing the entries in Table 1 , write (6) in terms of the $t$-evolution of $\alpha_{i}$ 's and $Y_{i}^{\prime}$ 's, where $\alpha_{i}=g_{i}^{2} /(4 \pi)$ and 


$$
\begin{aligned}
& Y_{i}=h_{i}^{2} /(4 \pi), Y_{i j k}=\lambda_{i j k}^{\prime \prime 2} /(4 \pi) . \text { Thus } \\
& \frac{d \alpha_{Y}}{d t}=\left(2 n_{f}+\frac{3}{5}\right) \alpha_{Y}^{2}, \\
& \frac{d \alpha_{2}}{d t}=\left(-6+2 n_{f}+1\right) \alpha_{2}^{2}, \\
& \frac{d \alpha_{3}}{d t}=\left(-9+2 n_{f}\right) \alpha_{3}^{2}, \\
& \frac{d Y_{\tau}}{d t}=\left(4 Y_{\tau}+3 Y_{b}+3 \alpha_{2}-\frac{9}{5} \alpha_{Y}\right) Y_{\tau}, \\
& \frac{d Y_{b}}{d t}=\left(6 Y_{b}+Y_{t}+Y_{\tau}+6 Y_{\not B}-\frac{16}{3} \alpha_{3}-3 \alpha_{2}-\frac{7}{15} \alpha_{Y}\right) Y_{b}, \\
& \frac{d Y_{t}}{d t}=\left(6 Y_{t}+Y_{b}+6 Y_{\not B}-\frac{16}{3} \alpha_{3}-\frac{13}{15} \alpha_{Y}\right) Y_{t}, \\
& \frac{d Y_{B}}{d t}=\left(2 Y_{b}+2 Y_{t}+18 Y_{B}-8 \alpha_{3}-\frac{4}{5} \alpha_{Y}\right) Y_{\not B} .
\end{aligned}
$$

In (7), $n_{f}$ is the number of generations and we have defined $Y_{\not B}$ as the sum $Y_{133}+Y_{233}$ for convenience.

The low energy constraints on $Y_{b}, Y_{t}$ and $Y_{\tau}$ come from the following relations:

$$
\begin{aligned}
& \sqrt{4 \pi Y_{t}\left(m_{t}\right)}=m_{t}\left(m_{t}\right) \sqrt{1+\tan ^{2} \beta}(174 \mathrm{GeV} \tan \beta)^{-1} \\
& \sqrt{4 \pi Y_{b}\left(m_{t}\right)}=m_{b}\left(m_{t}\right) \sqrt{1+\tan ^{2} \beta}\left(174 \mathrm{GeV} \eta_{b}\right)^{-1} \\
& \sqrt{4 \pi Y_{\tau}\left(m_{t}\right)}=m_{\tau}\left(m_{\tau}\right) \sqrt{1+\tan ^{2} \beta}\left(174 \mathrm{GeV} \eta_{\tau}\right)^{-1}
\end{aligned}
$$

In (8) $m_{t}\left(m_{t}\right), m_{b}\left(m_{b}\right), m_{\tau}\left(m_{\tau}\right)$ are the top, bottom and tau masses at $\mu=m_{t}, \mu=m_{b}, \mu=m_{\tau}$ respectively. We use $m_{b}\left(m_{b}\right)=4.25 \pm .15 \mathrm{GeV}$ and $m_{\tau}\left(m_{\tau}\right)=1.777 \mathrm{GeV}$ as input values. Moreover, with running effects from loops taken into account [9], one may take $\eta_{b} \equiv m_{b}\left(m_{t}\right) / m_{b}\left(m_{b}\right)=1.54$ for $\alpha_{s}=.123 \pm .004$ and $\eta_{\tau} \equiv m_{\tau}\left(m_{t}\right) / m_{\tau}\left(m_{\tau}\right) \simeq 1$. In our notation, $\tan \beta$ is the ratio of vacuum expectation value of $H_{2}$ to that of $H_{1}$. We know from 
phenomenological analyses that $[11] \tan \beta$ lies between 1 and $m_{t} / m_{b}$. Once the values of $Y_{t}, Y_{b}$ and $Y_{\tau}$ are fixed at the scale $m_{t}$, they can be evolved to the scale of SUSY breaking $M_{S U S Y}$ using non-SUSY RGE equations. In our calculations we have used $M_{S U S Y}$ to be $1 \mathrm{TeV}$ since this is preferred by the data on gauge couplings for a unification scenario [2]. The top quark mass range is taken to be $125-185 \mathrm{GeV}$.

We have not assumed any boundary conditions on the Yukawa couplings at the unification scale. This makes our analysis independent of models of Yukawa coupling unification. We only require that all Yukawa coupling strengths remain perturbative $\left(Y_{i}<1\right)$. (Later, we will see the results in terms of the fixed points of these strengths). In our analysis this condition, when imposed, yields numerical upper bounds on the magnitude of $\lambda_{\not \beta}$, where $\lambda_{\not B}^{2} /(4 \pi)=Y_{B \bar{B}}\left(\right.$ i.e. $\left.\left|\lambda_{B B}\right|>\left|\lambda_{133}^{\prime \prime}\right|,\left|\lambda_{233}^{\prime \prime}\right|\right)$ at the scale $M_{S U S Y}$. The results have been collected in Table 2 .

\begin{tabular}{|c|c|c|c|c|}
\multicolumn{5}{c|}{$m_{t}(\mathrm{GeV}) \rightarrow$} \\
\hline $\tan \beta$ & 125 & 145 & 165 & 185 \\
\hline 1 & & & & $Y_{t}$ \\
\hline 5 & .63 & .60 & .56 & diverges \\
\hline 10 & .63 & .60 & .56 & for any value \\
\hline 20 & .62 & .58 & .56 & of $Y_{133}+Y_{233}$ \\
\hline 40 & .62 & .58 & .56 & at the scale \\
\hline 50 & .59 & .59 & .53 & $M_{S U S Y}$ \\
\hline
\end{tabular}

Table 2 : Upper bounds on $\lambda_{\beta}$ as functions of $\tan \beta$ and $m_{t}$. 
We can highlight the following points regarding the above results.

1. Though we have taken $M_{S U S Y} \simeq 1 \mathrm{TeV}$, the value preferred for gauge coupling unification [2], our bounds are insensitive to the magnitude of the supersymmetry breaking scale. The choice $M_{S U S Y} \simeq m_{t}$ yields more or less the same bounds with some changes in the second decimal place.

2. An interesting point to note is that, towards higher values of the top mass, the top coupling $Y_{t}$ itself diverges in the high $t$ region and the upper bound on the baryon number violating couplings cannot be derived.

3. The constraint of perturbative unitarity at $M_{S U S Y}$ would directly imply that $Y_{B}\left(M_{S U S Y}\right)<1$, i.e. $\lambda_{B}\left(M_{S U S Y}\right)<3.54$. The dramatic effect of RGE and of the constraint $Y_{B}(\mu)<1$ for $\mu<2 \times 10^{16} \mathrm{GeV}$ is to reduce this upper bound from 3.54 to $0.5-0.6$. Thus our achievement here, after using RGE, is the reduction of the naive upper bound by a factor of six or seven.

4. One can try to introduce a further restriction by requiring [12] the unification of the three baryon number conserving Yukawa couplings. Here we note that the $B$ Yukawa terms in the superpotential have a different matter parity from the $B$ conserving ones. Therefore, even at the unification scale, there is no reason to expect the equality of the $B$-conserving and $B$ couplings. However, the requirement of the 
unification of $Y_{t}, Y_{b}$ and $Y_{\tau}$ at that high scale mainly pushes the value of $\tan \beta$ towards the higher region. We see from Table 2 and Fig.1 that these bounds are contained in our result. In fact, the upper bounds do not vary much with $\tan \beta$ when the latter is large. However, $Y_{b}$ and $Y_{\tau}$ start contributing substantially to the evolution of $Y_{B}$ for higher values of $\tan \beta$. This reduces the upper bound somewhat.

5. Ours is only a 1-loop RGE analysis. Moreover, we have not included the threshold effects here since they are typically of the order of 2-loop terms [13] which have been ignored. Since ours is the initial investigation in this direction, we have tried to be approximate and simple, rather than very accurate and complicated.

There have been a number of studies [14] on the fixed point behaviour of the top quark Yukawa coupling $Y_{t}$ in the MSSM. It has been found from the RGE that there is a fixed point value for $Y_{t}\left(m_{t}\right)$ starting from a large range of $Y_{t^{-}}$-values at the unification scale. With $\not B, \not R_{p}$ couplings present however, two questions arise automatically:

- What happens to the fixed point of $Y_{t}$ now?

- Is there a fixed point in $Y_{B}$ ?

We find that the evolutions of $Y_{t}$ and $Y_{B P}$ are mutually dependent in a way that the fixed point in $Y_{t}$ is reduced from the value it has in the MSSM. First, 
we have calculated the approximate fixed point of the Yukawa couplings by taking arbitrarily large values of $Y_{t}$ and $Y_{\not B}$ at the unification scale. Such a scenario is possible in the $\mathrm{SU}(5)$ GUT where, at the unification scale, one has the relation $h_{\tau}=h_{b} \neq h_{t}$. It turns out that, in the presence of $B$-violating Yukawa couplings, the fixed point of $h_{t}$ reduces from 1.06 in the MSSM to 0.88. We simultaneously get a fixed point value of the $\not B$ coupling $\lambda_{\not}$ around 0.59. On the other hand, we can make all $Y$ 's including $Y_{\tau}$ and $Y_{b}$ arbitrarily large at the unification scale. This scenario may appear in the $\mathrm{SO}(10)$ GUT in the presence of a $16 \times 16 \times 10$ coupling at the GUT scale. In this case the fixed point value of $h_{t}$ reduces from 1.0 in the MSSM to nearly 0.86 in the presence of $B$-violation while the fixed point value of $\lambda_{B}$ is about 0.65 .

Let us summarize our conclusions regarding constraints on supersymmetric $B$-violating couplings. Upper bounds have been derived on the magnitudes of the generation-hierarchically largest B Yukawa couplings, possible in the MSSM extended to include $R_{p^{-}}$and $B$-violation, at the SUSY breaking scale. These are not very strong but are the first acceptable bounds on these hitherto unresticted couplings. Moreover, the present work treats the evolution of $R_{p}, \not B$ coupling strengths and their fixed point behavior in relation to that of the top Yukawa coupling. These bounds have emerged from the requirement of the validity of perturbative behavior at all energies below the unification scale. Similar considerations can also be applied to the other viable $\not R_{P}$ model with the violation of lepton (but not baryon) number. However, in that case the quadratic self-driving term would be smaller due 
to the absence of any color factor in the 1-loop graphs containing leptons. So we expect less strong constraints there.

B.B. would like to acknowledge the hospitality of the Tata Institute of Fundamental Research and thank Prof. D.P. Roy for an initial suggestion towards this work. P.R. would like to acknowledge the hospitality of the Physical Research Laboratory. This paper was completed at the workshop WHEPP3 (January 10-22, 1994) held at the Institute of Mathematical Sciences, Madras, with joint sponsorship by the S.N. Bose National Centre for Basic Sciences. 


\section{References}

[1] L.J. Hall and M. Suzuki, Nucl. Phys. B231, 419 (1984), S. Dawson, Nucl. Phys. B261, 297 (1985), V. Barger, G.F. Giudice, T. Han, Phys. Rev. D40, 2987 (1989). L.J. Hall, Mod. Phys. Lett. A5, 467 (1990).

[2] U. Amaldi et. al., Phys. Lett. B260, 447 (1991).

[3] H.P. Nilles, Phys. Rep. C110, 1 (1984). P. Nath, R. Arnowitt and A.H. Chamseddine, Applied $N=1$ supergravity (World Scientific, Singapore, 1984). H.E. Haber and G.L. Kane, Phys. Rep. C117, 75 (1985).

[4] D. Brahm and L.J. Hall, Phys. Rev. D40, 2449 (1989), L.E. Ibáñez and G.G. Ross, Nucl. Phys. B292, 400 (1987).

[5] R. Barbieri and A. Masiero, Nucl. Phys. B267, 679 (1986). A. Bouquet and P. Salati, Nucl. Phys. B284, 557 (1987). B.A. Campbell, S. Davidson, J. Ellis and K. Olive, Phys. Lett. B256, 457 (1991). J. Ellis and K. Olive, Phys. Lett. B256, 457 (1991). E. Roulet and D. Tommasini, Phys. Lett. B256, 218 (1991). W. Fischler, G. Giudice, R.G. Leigh and S. Paban, Phys. Lett. B258, 45 (1991). A. Nelson and S.M. Barr, Phys. Lett. B258, 45 (1991).

[6] H. Dreiner and G.G. Ross, Nucl. Phys. B410, 188 (1993).

[7] V. Barger et al, Ref. 1. R. Godbole, P. Roy and X. Tata, Nucl. Phys. B401, 67 (1993). 
[8] F. Zwirner, Phys. Lett. B132, 103 (1983).

[9] P. West, "Introduction to supersymmetry and supergravity" (World Scientific, Singapore, 1990).

[10] V. Barger, M.S. Berger and P. Ohmann, Phys. Rev. D47, 1093 (1993).

[11] Z. Kunszt and F. Zwirner, Nucl. Phys. B385, 3 (1992).

[12] B. Ananthanarayan, G. Lazarides, Q. Shafi, Phys. Rev. D44, 1613 (1991).

[13] J. Ellis, S. Kelley, D.V. Nanopoulos, Nucl. Phys. B373, 55 (1992); P. Langacker and N. Polonsky, Phys. Rev. D47, 4028 (1993).

[14] B. Pendleton and G. G. Ross, Phys Lett B98 291 (1981); C. T. Hill, Phys. Rev D24, 691, (1981). For a brief summary of RGE results for SUSY GUTS see V. Barger, M. S. Berger, P. Ohmann and R. N. J. Phillips, University of Wisconsin Madison report, MAD/PH/803 and references therein. 


\section{Figure caption}

Fig. 1 Three curves showing the variation on the upper bound on $\lambda_{\beta}$ with respect to the free parameter $\tan \beta$ for three different values (in $\mathrm{GeV}$ ) of the top mass. 
This figure "fig1-1.png" is available in "png" format from: http://arxiv.org/ps/hep-ph/9403350v2 